\title{
MONOTONY OF THE OSCULATING CIRCLES OF ARCS OF CYCLIC ORDER THREE
}

\author{
N. D. Lane, K. D. Singh and P. Scherk
}

(received November 1, 1963)

1. Introduction. It is well-known in elementary calculus that if a differentiable function has a monotone increasing curvature, then its curvature is continuous and the circles of curvature at distinct points have no points in common. In particular, two one-sided osculating circles at distinct points of an arc $A_{3}$ of cyclic order three have no points in common; cf. [1], [2], [3]. The conformal proof given here that any two general osculating circles at distinct points of $\mathrm{A}_{3}$ are disjoint (Theorem 1), may be of interest. We also prove that all but a countable number of points of $\mathrm{A}_{3}$ are strongly conformally differentiable (Theorem 2).

2. The notations and definitions used in this discussion are the same as in [4] and [5]. For the convenience of the reader, we list some of the results which are needed here.

An arc $A$ in the conformal plane is the continuous image of a real interval. $P, Q, \ldots$ denote points in the conformal plane, and $p, s, q, \ldots$ denote points of arcs. C denotes an oriented circle, with the interior $C_{*}$ and exterior $C^{*}$, the latter region lying at its right.

An arc $A$ is called once conformally differentiable at $p$ if it satisfies the following:

CONDITION I. There exists a point $Q \neq p$ such that if $s$ is sufficiently close to $P$ on $A$, then the circle $C(p, s, Q)$ exists. It converges if $s$ converges to $P$ [4; Theorem 1].

Canad. Math. Bull. vol. 7, no.2, April 1964 
We denote the limit tangent circle by $C(\tau ; Q)$.

If Condition I holds for a single point $Q \neq p$, then it holds for all such points, and the closed set $\tau=\tau(p)$, of all the tangent circles of $A$ at $p$ is a parabolic pencil, i.e., any two circles of $\tau$ meet at $p$ and nowhere else.

We call A conformally differentiable at $p$ if it satisfies

CONDITION II. If $s \neq p$, then $\lim _{s \rightarrow p} C(\tau ; s)$ exists.

The limit osculating circle is denoted by $C(p)$.

We call $C$ a general tangent circle of an arc $A$ at $p$, if there exists a sequence of triples of mutually distinct points $t_{n}, u_{n}, Q_{n}$, such that $t_{n}$ and $u_{n}$ converge on $A$ to $p$, and $\lim C\left(t_{n}, u_{n}, Q_{n}\right)=C$. If, in addition, $Q_{n} \in A$ also converges to $\mathrm{p}$, then we call $\mathrm{C}$ a general osculating circle of $\mathrm{A}$ at $\mathrm{p}$.

$\mathrm{A}_{3}$ denotes an arc of cyclic order three; thus no circle meets $A_{3}$ more than three times. Here, $p$ is counted twice on any general tangent circle of $A$ at $p$ which is not a general osculating circle. On a general osculating circle, and, in particular, on $C(p), p$ is counted three times; cf. [5; Section 3].

Each point of $\mathrm{A}_{3}$ has the property that if $Q, R \neq p$, $\mathrm{Q} \rightarrow \mathrm{R}$ and two distinct points $\mathrm{u}$ and $\mathrm{v}$ converge on $\mathrm{A}_{3}$ to $\mathrm{p}$, then $C(u, v, Q)$ always converges [5; Theorem 2].

If $\mathrm{p}$ is an end-point of $\mathrm{A}_{3}$, then $\mathrm{C}(\mathrm{t}, \mathrm{u}, \mathrm{v})$ converges if the three mutually distinct points $t, u, v$ converge on $A$ to $p$ [5; Theorem 3].

3. Let $p \in A_{3}$. Let $B_{3}$ denote the open subarc of $A_{3}$ bounded by $P$ and an end-point of $A_{3}$. Let $C$ be any general osculating circle of $A_{3}$ at $p$, and let $C(p)$ be the (unique) osculating circle of $\mathrm{B}_{3}$ at $\mathrm{p}$. 
If $\mathrm{p}$ is an end-point of $\mathrm{A}_{3}$, the strong differentiability of $\mathrm{A}_{3}$ at $\mathrm{p}$ implies that $\mathrm{C}=\mathrm{C}(\mathrm{p})$ (cf. [5], Theorem 3).

Suppose, next, that $\mathrm{p}$ is an interior point of $\mathrm{A}_{3}$. Then $C$ and $C(p)$ both intersect $A_{3}$ at $p$ (cf. [5], Section 3. 3). By [5; Theorem 2], the general tangent circles of $\mathrm{A}_{3}$ at $p$ form a pencil $T$; thus, $C \in T, C(F) \in \tau$.

LEMMA. If $C^{*} \mathrm{C} C(\mathrm{p})^{*}$, then $\mathrm{B}_{3} \mathrm{C} \mathrm{C}(\mathrm{p})_{*}$.

Proof. By [5; Sections 3.32 and 3.33], $B_{3} \cap C=B_{3} \cap C(p)=p$. Suppose that $\mathrm{B}_{3} \mathrm{CC}(\mathrm{p})^{*}$. Then $\mathrm{B}_{3} \mathrm{CC}(\mathrm{p})^{*} \cap \mathrm{C}_{*}$; otherwise, $C(\tau ; s)$ could not converge to $C(p)$ as $s$ tends to $p$ on $B_{3}$. This implies, however, that $C(p)$ and $C$ cannot both intersect $\mathrm{A}_{3}$ at $\mathrm{p}$.

COROLLARY. If $\mathrm{p}$ is an interior point of $\mathrm{A}_{3}$, then any general osculating circle of $\mathrm{A}_{3}$ at $\mathrm{p}$ lies between the two onesided osculating circles of $A_{3}$ at $p$ in the pencil $T(p)$ (cf. [5], 3. 42).

4. THEOREM 1. Two general osculating circles at distinct points of $\mathrm{A}_{3}$ have no points in common.

Proof. On account of the above Corollary, we may now assume that $A_{3}$ is an open arc with the end-points $p$ and $q$. Thus, $A_{3}$ has uniquely defined osculating circles $C(p)$ and $C(q)$ at $p$ and $q$, respectively. We may assume that neither $C(p)$ nor $C(q)$ is a point-circle. Let $\tau$ and $\tau$ denote the families of tangent circles at $p$ and $q$, respectively.

If $t, u, v$ lie on $\mathrm{A}_{3}$ in that order, we may assign to $C(t, u, v)$ the orientation associated with the order of the points $t, u, v$ on $C(t, u, v)$. 
Thus, the arc $\mathrm{A}_{3}$ induces a natural and continuous

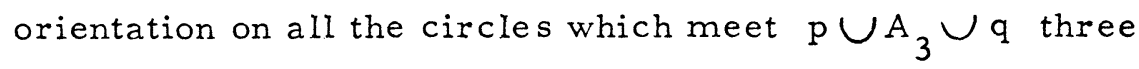
times (cf. [5], Section 3.51).

We may assume that $\mathrm{A}_{3} \mathrm{C} C(\mathrm{p})_{*}$. By considering the circles $C(\tau: s)$ and $C(p, s, q)$, and letting $s$ move from $p$ to $q$ on $A_{3}$, we readily verify that

$$
\mathrm{A}_{3} \mathrm{CC}(\mathrm{p})_{*} \cap \mathrm{C}(\tau ; \mathrm{q})^{*} \cap \mathrm{C}\left(\mathrm{p} ; \tau_{\mathrm{q}}\right)_{*} \cap \mathrm{C}(\mathrm{q})^{*} \text {, }
$$

$$
C(\tau ; q)_{*} C C(p)_{*} \text {, and } C\left(p ; \tau_{q}\right)^{*} C C(q)^{*}
$$

Since $C\left(p ; \tau_{q}\right) \neq C(\tau ; q), C\left(p ; \tau_{q}\right)$ intersects $C(\tau ; q)$ at $p$ and $q$. Hence $C\left(p ; \tau_{q}\right)$ also intersects $C(p)$ at $p$ and at another point. Since $C(\tau ; q)$ intersects $C\left(p ; \tau q^{\prime}\right.$ at $q$, $C(\tau ; q)$ also intersects $C(q)$ at $q$. Thus $C(\tau ; q)$ and $C(q)$ intersect at another point $R$. The points $q$ and $R$ decompose $C(q)$ into two arcs $C^{\prime}$ and $C^{\prime \prime}$, such that $C^{\prime} C C\left(p ; \tau_{q}\right)_{*} \cap C(\tau ; q)_{*}$, while $C^{\prime \prime} C \mathrm{C}\left(\mathrm{p} ; \tau_{\mathrm{q}}\right)_{*} \cap \mathrm{C}(\tau ; q)^{*}$. Since $\left.\mathrm{C}(\tau ; q)\right)_{*} C \mathrm{C}(\mathrm{p})_{\text {* }_{k}}$, we obtain $\mathrm{C}^{\prime} \mathrm{C}(\mathrm{p})_{*}$.

Suppose that $C^{\prime \prime}$ meets $C(p)$; thus $C^{\prime \prime}$ meets $C(p) \cap C\left(p ; \tau_{q}\right)_{*} \cdot$ Then $C^{\prime \prime}$ decomposes the region

$$
\mathrm{C}(\mathrm{p})_{2_{k}} \cap \mathrm{C}\left(\mathrm{p} ; \mathrm{T}_{\mathrm{q}}\right)_{\psi_{k}} \cap \mathrm{C}(\mathrm{\tau} ; \mathrm{q})^{*}
$$

into three disjoint regions. Two of these lie in

$$
C\left(p ; \tau_{q}\right)_{*} \cap C(q)^{*} \cap C(p)_{*}
$$

and their boundaries have at most a single point in common which lies in $C(p)$. The region of (2) whose boundary includes an arc of $\mathrm{C}(\tau ; q)\left[\mathrm{C}\left(\mathrm{p} ; \tau_{\mathrm{q}}\right)\right]$ contains points of $\mathrm{A}_{3}$ close to $\mathrm{p}[\mathrm{q}]$. But then the continuity of $\mathrm{A}_{3}$ and Relation (1) imply 
that the se two regions are connected. Hence $C^{\prime \prime} C \mathrm{C}(\mathrm{p})_{*}$, and the whole of $\mathrm{C}(\mathrm{q})=\mathrm{C}^{\prime} \cup \mathrm{C}^{\prime \prime} \cup\{\mathrm{q}, \mathrm{R}\}$ lies in $\mathrm{C}(\mathrm{p})_{\star}$.

Remark. The following alternative method of proving that $\overline{C^{\prime \prime} C \mathrm{C}(\mathrm{p})_{*}}$ is shorter and direct, but it requires the full Jordan curve theorem.

As above, $C^{\prime \prime} \mathrm{C} \mathrm{C}\left(\mathrm{p} ; \tau_{\mathrm{q}}\right)_{*} \cap \mathrm{C}(\tau ; \mathrm{q})^{*}$. Since $\mathrm{C}(\mathrm{q})$ does not meet $A_{3}, C^{\prime \prime}$ even lies in the region in $C\left(p ; \tau_{q}\right)_{*}$ bounded by $\mathrm{A}_{3}$ and $\mathrm{C}(\tau ; q)$. Hence $\mathrm{C}^{\prime \prime} \mathrm{C} C(\mathrm{p})_{*}$.

5. THEOREM 2. All but a countable number of points of $\mathrm{A}_{3}$ are strongly conformally differentiable; cf. [6].

Proof. Let $\mathrm{p}$ and $\mathrm{q}$ be the end-points of $\mathrm{A}_{3}$. We may as sume that $\mathrm{C}(\mathrm{p}) \neq \mathrm{p}$, and $\mathrm{A}_{3} \mathrm{C} C(\mathrm{p})_{*}$. By choosing a suitable co-ordinate system we may even assume that $C(p)$ is a circle of area 1.

Let $s \in \mathrm{A}_{3}$ be a point at which $\mathrm{A}_{3}$ is not strongly conformally differentiable; then $\mathrm{A}_{3}$ does not satisfy Condition II at $s$; cf. 3, Corollary. Let $C(s)$ and $C^{\prime}(s)$ be the onesided osculating circles of $\mathrm{A}_{3}$ at $\mathrm{s}$. We may assume that $C(s)_{*} C^{\prime}(s)_{*}$. Let $f(s)$ be the area between $C(s)$ and $C^{\prime}(s)$. By Theorem 1, the regions $C(s)^{*} \cap C^{\prime}(s)_{*}$ and $C(t)^{*} \cap C^{\prime}(t)_{*}$ are disjoint if $s \neq t$, and they lie in $C(p)_{*}$.

Thus there are not more than $2^{\mathrm{n}}$ members in the class of points $s$ for which

$$
1 / 2^{n-1}>f(s) \geq 1 / 2^{n} \quad(n=1,2,3, \ldots) .
$$

Since every point $s \in A_{3}$ with $f(s)>0$ is included in exactly one of these classes, there is only a countable set of points $s$ with $f(s)>0$. 


\section{REFERENCES}

1. J. Hjelmslev, Die graphische Geometrie, Attönde Skandinav. Mat-Kong. Fortand 1 (Stockholm, 1934).

2. H. Haller, Ueber die $\mathrm{K}_{3}$-Schmieggebilde der ebenen Bogen von der $\mathrm{K}_{3}$-Ordnung drei, S. -B. Phys.-Med. Soz. Erlangen, 69 (1937), 15-18.

3. O. Haupt, Zur geometrischen Kennzeichnung der Scheitel ebener Kurven, Archiv der Mathematik (1948), 102-105.

4. N.D. Lane and Peter Scherk, Differentiable points in the conformal plane, Can. J. Math. 5 (1953), 512-518.

5. , Characteristic and order of differentiable points in the conformal plane, Trans. Amer. Math. Soc., 81 (1956), 353-378.

6. A. Marchaud, Sur les continus d'ordre borné, Acta Math. 55 (1930), 57-115.

\footnotetext{
McMaster University and University of Toronto
} 


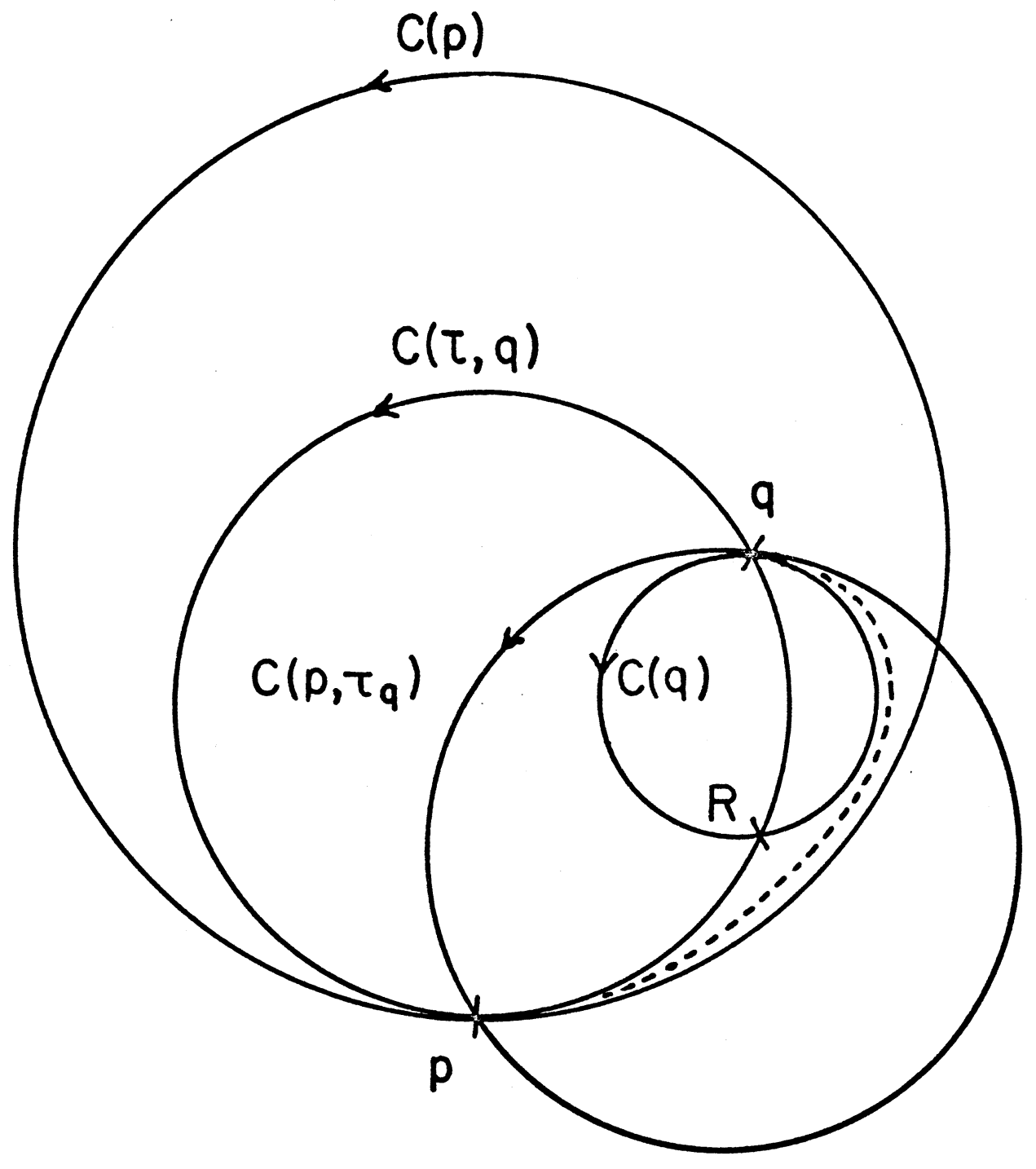

